\title{
Minimizing the Disaster Risk in Optical Telecom Networks
}

\author{
"Ferhat Dikbiyik ${ }^{1}$, Abu S. Reaz ${ }^{2}$, Marc De Leenheer ${ }^{3}$ and Biswanath Mukherjee ${ }^{4}$ \\ ${ }^{1}$ Department of Electrical \& Computer Engineering, University of California, Davis, CA 95616 \\ 2 Park, Vaughan, Fleming \& Dowler LLP, Davis, CA 95618 \\ ${ }^{3}$ Department of Information Technology, Ghent University-IBBT, Ghent, Belgium 9000 \\ ${ }^{4}$ Department of Computer Science, University of California, Davis, CA 95616 \\ *Corresponding author:fdikbiyik@ucdavis.edu
}

\begin{abstract}
Regional failures caused by disasters precipitate huge amount of loss and jeopardize many connections. A risk model for disasters, considering differentiated services, is introduced and a disaster-aware provisioning scheme is proposed to reduce the risk. (C)2012 Optical Society of America

OCIS codes: (060.4256) Networks, network optimization; (060.4257) Networks, network survivability
\end{abstract}

\section{Introduction ${ }^{1}$}

Regional failures, where multiple network elements in a region fail simultaneously, are usually caused by disasters such as earthquakes, hurricanes, weapons of mass destruction (WMD), etc. [1]. Such disasters precipitate huge amount of loss in optical telecom networks. For instance, in 2008 Shicuan Earthquake in China, 30,000 km of fiber cables and 4,000 of telecom offices were damaged [2]. Several studies consider such regional/disaster failures (e.g., [1], [3-5]), and aim to find the vulnerable parts of the network to disasters for analysis and/or design purposes. How to provision connections with the knowledge of vulnerable regions is a traffic engineering problem and we focus on this problem in this study. We explore the risk of traversing through these vulnerable parts in an optical network.

In statistics, risk is defined as the expected value of some outcome seen as undesirable. Ref. [6] proposes such risk model for transportation network in case of an earthquake. We propose a similar risk analysis for optical telecom networks in terms of penalty paid by network operator (NO) to the customers. This penalty is usually stated in Service Level Agreement (SLA) and expressed in terms of penalty per unit time when the down time exceeds allowed down time (ADT). We also discuss the probability of damage, probability of disaster, and number of possible events which should be taken into consideration.

In this study, our contribution is two-fold. First, we develop a probabilistic risk model to analyze the loss/penalty given the set of possible disasters. Second, we provide a traffic engineering solution for disaster protection by proposing a disaster risk-aware provisioning in optical telecom networks where we develop a mathematical model which reduces the risk and decreases the loss/penalty in case of a disaster. We formulate the problem into an Integer Linear Program (ILP). The illustrative numerical examples show that our approach reduces the risk by $6.5 \%$ with a slight increase in resources. We also provide a penalty model to compute the loss of NO in case of a disaster.

\section{Risk and Penalty Model}

We define the risk in Eq. (1) which captures the basic elements of risk analysis [7]: i) what can happen? ii) How likely is it that that will happen? iii) If it does happen, what are the consequences?

$$
R=\sum_{n \in N}\left(\sum_{t \in T} c_{t} Z_{t}^{n}\right) p_{d}^{n} p_{n}
$$

where $N$ is the set of possible disasters, $T$ is the set of connections, $c_{t}$ is the cost of losing connection $t, Z_{t}^{n}$ is equal to 1 if connection $t$ is down in case of disaster $n, p_{d}{ }^{n}$ is the probability of damage caused by disaster $n$, and $p_{n}$ is the probability of disaster $n$. The term in parenthesis gives the loss if disaster $n$ happens. $c_{t}$ gives us the benefit of differentiating between connections (e.g., some connections might be more valuable to lose than other connections).

We provide the following penalty model which NO should pay in case of a disaster. Let $h_{r}$ and $h_{\text {adt }}^{t}$ denote the average recovery time from a disaster (which might take weeks) and ADT of connection $t$ which is specified in SLA. The penalty in case of disaster $n$ can be formulated as

$$
P_{n}=\sum_{t \in T} c_{t} Z_{t}^{n}\left(h_{r}-h_{a d t}^{t}\right)
$$

\footnotetext{
${ }^{1}$ This work has been supported by the Defense Threat Reduction Agency (DTRA) Program "Network Adaptability from WMD Disruption and Cascading Failures".
} 


\section{Disaster Risk-Aware Provisioning (D-RAP)}

Our objective in provisioning a set of connection requests (with knowledge of set of possible disasters with their probability and possible damages) is minimizing the risk in Eq. (1) so that we can minimize the penalty in case of a disaster. We formally state the problem of disaster risk-aware provisioning and formulate the problem into an ILP approach for $1+1$ dedicated protection (where connections are routed over a primary path and a dedicated linkdisjoint backup path) as shown below. Note that equations can be modified easily for other protection schemes.

Given:

- $G(V, E)$ : Network topology.

- $T=\left\{t=\left\langle s_{t}, d_{t}, c_{t}\right\rangle\right\}$ : Set of connections with source $\left(s_{t}\right)$, destination $\left(d_{t}\right)$, and cost of losing connection $\left(c_{t}\right)$.

- $W_{i j}$ : Number of channels on link $(i, j)$

- $N=\left\{n=\left\langle L_{n}, p_{n}, p_{d}{ }^{n}\right\rangle\right\}$ : set of disasters with $L_{n}\left(\left|L_{n}\right|=|E|\right)$ is the set of binary values $l_{n}^{i j}$ (which is 1 if link ( $i$, $j$ ) is disconnected by disaster $n$, and 0 otherwise), probability of disaster $n\left(p_{n}\right)$, probability of damage by disaster $n\left(p_{d}{ }^{n}\right)$.

Binary variables:

- $R_{t}^{n}\left(B_{t}{ }^{n}\right)=1$ if the primary (backup) path of connection $t$ is down in case of event $n$, otherwise 0 .

- $R_{i j}{ }^{t}\left(B_{i j}{ }^{t}\right)=1$ if the primary (backup) path of connection $t$ traverses link $(i, j)$, otherwise 0 .

- $Z_{t}^{n}=1$ if connection $t$ (on both primary and backup) is down in case of event $n$, otherwise 0 .

Constraints:

$$
\begin{array}{lll}
\sum_{t}^{n} \geq \frac{\sum_{(i, j) \in E} l_{n}^{i j} R_{i j}^{t}}{M} \quad \forall(i, j) \in E, t \in T, n \in N & & R_{t}^{n} \leq \sum_{(i, j) \in E} l_{n}^{i j} R_{i j}^{t} \quad \forall(i, j) \in E, t \in T, n \in N \\
Z_{t}^{n} \leq R_{t}^{n} \quad t \in T, n \in N & \text { (3) } & Z_{t}^{n} \geq R_{t}^{n}+B_{t}^{n}-1 \quad t \in T, n \in N \\
R_{i j}^{t}+X_{i j}^{t}+R_{j i}^{t}+X_{j i}^{t} \leq 1 \quad \forall(i, j) \in E, \forall t \in T & \text { (5) } & \sum_{t \in T}\left(R_{i j}^{t}+B_{i j}^{t}\right) \leq W_{i j} \quad \forall(i, j) \in E, \forall t \in T
\end{array}
$$

Eq. (3)-(5) are for primary path and equations for backup path can be written by replacing $R$ by $B$. Eq. (3)-(6) are required to make sure that $Z_{t}^{n}$ is 1 if both primary and backup path of connection $t$ are disconnected by disaster $n$. Eq. (7) is required to make sure that primary and backup path are disjoint and Eq. (8) is link-capacity constraint. We also have flow-conservation constraints for both primary and backup paths where the equations are omitted due to the space limitation.

\section{Probability of Disaster and Probability of Damage}

The probability of a disaster and probability of damage are disaster-dependent. Here, we give two examples: a natural disaster (earthquake) and a man-made disaster (weapons of mass destruction (WMD) attacks). For an earthquake, seismic hazard maps indicate the probability of an earthquake and show different seismic hazard levels. The probability of damage depends on these seismic hazard levels. In [8], Grünthal explains how to provide damage curves for different seismic hazard levels. By using seismic hazard maps and damage curves, we can obtain the probability of damage of different hazard levels which indicates on which regions we should focus. For WMD attack, we can estimate the possible targets (populated cities, capitals, etc.) and we can have probability of attack proportional to the importance and/or population of target. The probability of damage, on the other hand, is close to 1 since these attacks are very destructive and intense.

\section{Illustrative Numerical Examples}

We show some illustrative numerical examples on US-wide Wavelength Division Multiplexing (WDM) network (NSFNet) shown in Fig. 1 with 32 wavelengths/link in each direction with wavelength conversion. In Fig. 1, we match physical NSFNet topology (obtained by matching NSFNet with transportation map where we assume that fiber links are close to highways since they should be accessible in case of a failure) with US seismic hazard map [9] with $2 \%$ probability of exceedance in 50 years $\left(p_{n}=0.02\right)$. We compute the probability of damage for different hazard levels and we neglect the hazard levels below $32 \%$ because of limited to no damage. Ref. [10] shows us that the damage of an earthquake may span up to $96 \mathrm{~km}$. Thus, we draw a circle with $96 \mathrm{~km}$ radius and circulate it in the considered areas; we obtain 11 distinct shared risk groups (shown in Fig. 1). We also consider that an earthquake on a fault line can trigger other earthquakes on the same line (i.e., correlated failures). We obtain the traffic demand 
such that connection requests are proportional to the populations of source and destination [11]. We assign connections different $c_{t}$ values: $10 \alpha, 5 \alpha, 3 \alpha$, and $\alpha$ with the distribution 1:2:3: 4 where the number of connections with high penalty is less than the others. $\alpha$ is some value to parameterize the penalty (e.g., penalty per hour).

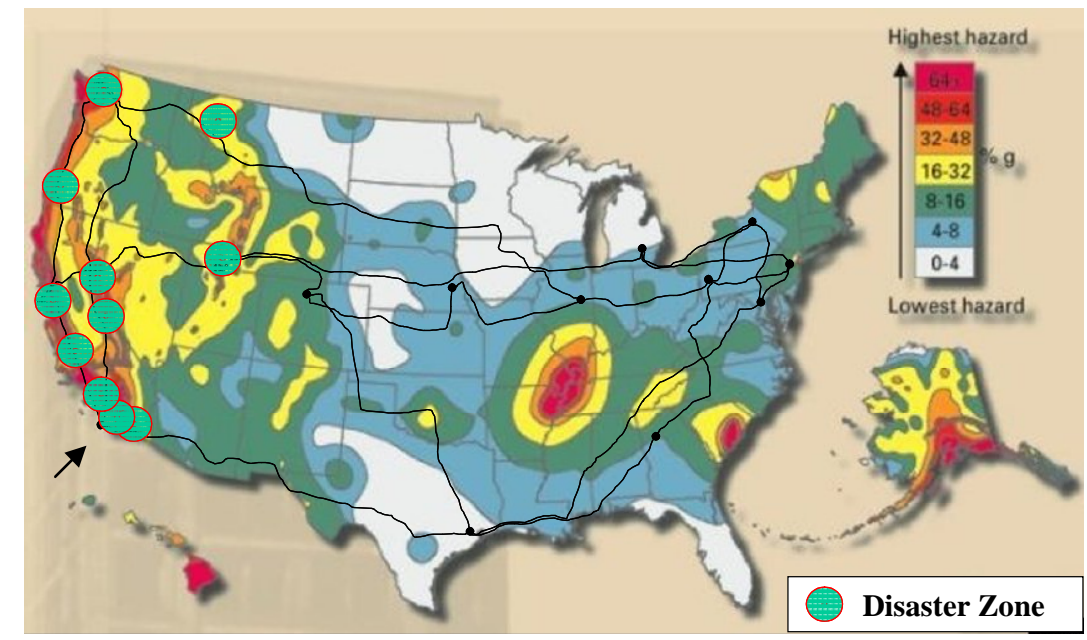

Figure 1. NSFNet representative physical topology and US seismic hazard map with disaster zones

Table I. Risk Reduction and Increase in Resources

\begin{tabular}{|c|c|c|c|c|c|c|c|c|c|}
\hline Initial Traffic Value (Tbps) [11] & 3 & 3.5 & 4 & 4.5 & 5 & 5.5 & 6 & 6.5 & 7 \\
\hline Risk Reduction & $5.57 \%$ & $4.60 \%$ & $6.59 \%$ & $2.72 \%$ & $6.29 \%$ & $4.04 \%$ & $4.67 \%$ & $6.38 \%$ & $3.74 \%$ \\
\hline Increase in Resources & $4.76 \%$ & $6.36 \%$ & $8.15 \%$ & $8.22 \%$ & $8.93 \%$ & $10.16 \%$ & $10.27 \%$ & $10.79 \%$ & $16.52 \%$ \\
\hline
\end{tabular}

We compare our approach, disaster risk-aware provisioning (D-RAP), to an approach which aims to minimize resource occupation with $1+1$ protection (where connections are provisioned over link-disjoint primary and backup path), which we called $\min R$. Table I shows risk reduction and increase in resources of $D$-RAP compared to $\min R$. Results show that our approach reduces the risk by up to $6.5 \%$. Risk reduction results in increasing in resources up to $16.5 \%$. We explore the penalty with a case study where we have an earthquake in San Diego area (shown with an arrow in Fig. 1) where the initial traffic value [11] to generate traffic is 7 Tbps. Assume that recovery time is one week after disaster $\left(h_{r}=168 \mathrm{hrs}\right.$.) and allowed down time for a connection is 2 days ( $\left.h_{\text {adt }}^{t}=48 \mathrm{hrs}\right)$. In this case, our approach decreases penalty paid by network operator by $4,140 \alpha$, where $\alpha$ is penalty per hour.

We also have illustrative numerical examples for WMD attacks where we obtain 5.5\% risk reduction with $20 \%$ increase in resources. We omit the details due to the space limitation.

\section{References:}

[1] S. Neumayer, G. Zussman, R. Cohen, and E. Modiano, "Assessing the Vulnerability of Fiber Infrastructure to Disasters," in Proc. IEEE INFOCOM, Brazil, April 2009.

[2] Y. Ran, "Considerations and Suggestions on Improvement of Communication Network Disaster Countermeasures after the Wenchuan Earthquake," IEEE Communication Magazine, vol. 49, no. 1, pp. 44-47, January 2011.

[3] P. K. Agarwal, A. Efrat, S. Ganjugunte, D. Hay, S. Sankararaman, and G. Zussman, "The Resilience of WDM Networks to Probabilistic Geographical Failures," in Proc. IEEE INFOCOM, Shangai, China, April 2011.

[4] X. Wang, X. Jiang, and A. Pattavina, "Assessing Network Vulnerability under Probabilistic Region Failure Model," in Proc. IEEE HPSR, Cartegana, Spain, July 2011.

[5] S. Banerjee, S. Shirazipourazad, and A. Sen, "Design and Analysis of Networks with Large Components in Presence of Region-Based Faults," in Proc. IEEE ICC, Kyoto, Japan, June 2011.

[6] S. E. Chang, M. Shinozuka, and J.E. Moore, "Probabilistic Eartquake Scenarios: Extending Risk Analysis Methodologies to Spatially Distributed Systems," Earthquake Spectra, vol. 16, no. 3, August 2000.

[7] S. Kaplan and B. J. Garrick, "On the Quantitative Definition of Risk," Risk Analysis, vol. 1, no. 1, 1981.

[8] G. Grünthal, "Europan Macroseismic Scale 1998 (EMS-98),"Cahiers du Centre Europeen de Geodynamique et de Seismologie, vol. 15, Luxembourg, 1998.

[9] US Geological Survey, "2008 Hazard Map,” <http://earthquake.usgs.gov/hazards/products/graphic2pct50.jpg>.

[10] T. L. Weems, "How far is far enough," Disaster Recovery Journal, vol. 16, no. 2, Spring 2003.

[11] M. Batayneh, D.A. Schupke, M. Hoffman, A. Kristaedter, and B. Mukherjee, "On Routing and Transmission-Range Determination of MultiBit-Rate Signals over Mixed-Line-Rate WDM Optical Networks for Carrier Ethernet," IEEE/ACM Transactions on Networking, 2011 